\title{
Dynamically Constrained Interpolation of the Sparsely Observed Suspended Sediment Concentrations in Both Space and Time: A Case Study in the Bohai Sea
}

\author{
XINYAN MAO AND DAOSHENG WANG \\ Physical Oceanography Laboratory, and Qingdao Collaborative Innovation Center of Marine Science and Technology, \\ Ocean University of China, and Laboratory for Regional Oceanography and Numerical Modeling, \\ Qingdao National Laboratory for Marine Science and Technology, Qingdao, China \\ JICAI ZHANG \\ Institute of Physical Oceanography, Ocean College, Zhejiang University, Zhoushan, China
}

\section{CHANGWEI BIAN}

Physical Oceanography Laboratory, and Qingdao Collaborative Innovation Center of Marine Science and Technology, Ocean University of China, and Qingdao National Laboratory for Marine Science and Technology, Qingdao, China

\author{
XIANQING LV \\ Physical Oceanography Laboratory, and Qingdao Collaborative Innovation Center of Marine Science and Technology, \\ Ocean University of China, and Laboratory for Regional Oceanography and Numerical Modeling, \\ Qingdao National Laboratory for Marine Science and Technology, Qingdao, China
}

(Manuscript received 30 August 2017, in final form 15 March 2018)

\begin{abstract}
The observed suspended sediment concentrations (SSCs) obtained from the water sampling are usually sparsely distributed in both space and time, which are traditionally applied just to calibrate other types of observations. In this study a dynamically constrained interpolation methodology (DCIM) is developed to interpolate these sparsely observed SSCs in the Bohai Sea. In this method the suspended sediment transport model is taken as dynamical constraints to interpolate the observations. Meanwhile, the interpolated results are optimized iteratively by adjusting the key model parameters using the adjoint method.

The DCIM is first verified using the synthetic observations produced by twin model runs. The modeling results reveal that this method is effective at interpolating the sparsely observed artificial SSCs, even when the observations are heavily contaminated by data noise. Then, the sparsely observed practical SSCs obtained from a large area survey in the Bohai Sea are interpolated using the DCIM. The interpolated results are verified by randomly selected independent observations. The discrepancies between the interpolated SSCs and the observations are significantly decreased. When all the observations are interpolated, the final interpolated SSCs captured a majority $(96.88 \%$ ) of observations with a factor of 2 and the correlation coefficient between the observed and interpolated SSCs is 0.98 . Besides, the interpolated results have presented the reasonable dynamical variations of SSCs in the space and time domains. The modeling results indicate that the DCIM is an effective tool for interpolating the sparsely observed SSCs in both space and time.
\end{abstract}

\section{Introduction}

The coastal ocean plays an important role in global material cycling (Wang et al. 2014). Suspended sediments in the coastal environment have a major influence

Corresponding author: Daosheng Wang, dswangouc@163.com; Jicai Zhang, jicai_zhang@163.com on water quality (Zhang et al. 2008), geomorphic evolution (Jia et al. 2006; Fan et al. 2014), nutrient dynamics (Mayer et al. 1998), and so on. Therefore, the distribution and transport of suspended sediments are central to understanding many coastal and estuarine processes (Holdaway et al. 1999).

Many measurement techniques, including acoustic (e.g., Wilson and Hay 2015), bottle (e.g., Attard et al. 
2014), pump (e.g., Black and Rosenberg 1994), focused beam reflectance (e.g., Law et al. 1997), laser diffraction (e.g., Agrawal et al. 2008), nuclear (e.g., Crickmore et al. 1990), optical backscatter (e.g., Sutherland et al. 2000), optical transmission (e.g., Campbell et al. 2005), and spectral reflectance (e.g., Chen et al. 1991), have been used to measure the suspended sediment concentrations (SSCs) with varying degrees of success, whose operating principles, advantages, and disadvantages were discussed in Wren et al. (2000). The bottle and pump are the traditional suspended sediment measurement techniques, while the others are the surrogate suspended sediment measuring techniques (Gray and Landers 2014). Some surrogate techniques can obtain continuous SSC measurements, whose technological advances have been presented in Gray and Gartner (2009) and Rai and Kumar (2015). However, the gravimetric analysis of the water samples obtained from traditional techniques represents the standard methodology to physically quantify suspended sediment (Pavanelli and Bigi 2005). Although the traditional bottle sampling has poor spatial and temporal resolution and needs time-consuming processes to obtain and analyze the water samples, the analytical results of the bottle sampling are the most reliable tools to estimate SSC and are essential to properly calibrate measurements of the various surrogates (Gray et al. 2002). As the bottle sampling is almost the only method to get the mass concentration of suspended sediment, it is usually implemented at several points in space and time to calibrate other types of samplers, but these sparsely observed SSCs cannot accurately figure out the complex spatial and temporal variations of SSCs, which are jointly determined by tidal currents, water depth, and wind effects (Gartner 2004). A suitable interpolation method can be useful to improve the utilization of these sparse observations.

As summarized in Yaremchuk and Sentchev (2013), the interpolation methods can be classified as the dynamically unconstrained interpolation methods and the dynamically constrained interpolation techniques. The former ones are statistical methods, such as optimal interpolation and the two-dimensional variational technique, which do not make full use of dynamical information for a given dataset (Hoteit et al. 2009); in addition, the latter ones combine dynamical constraints from numerical models with statistical information from observations, which will produce dynamically and statistically consistent estimates (Yaremchuk and Sentchev 2013). For the data observed spatially and temporally, spatiotemporal interpolation is necessary to estimate the unknown values at unsampled location-time pairs with a satisfying level of accuracy ( $\mathrm{Li}$ and Revesz 2004). There are two dynamically unconstrained ways for spatiotemporal interpolation, including the reduction method, which treats time as an independent dimension, and the extension method, which treats time as equivalent to a spatial dimension ( $\mathrm{Li}$ and Revesz 2002). For the sparse observations in both space and time, both the reduction method and the extension method are rarely applied. For example, Ge et al. (2010) found that the direct simple interpolation of the time discontinuous large area observations of sea surface temperature produced a false frontal phenomenon. Therefore, the dynamically constrained interpolation techniques are needed to interpolate the sparse observations in both space and time. Based on the adjoint data assimilation method, the dynamically constrained interpolation technique has been applied to study the shelf circulation by spatiotemporally interpolating the ADCP data acquired from a moving ship (Griffin and Thompson 1996). The data monitors the Kuroshio Extension with dynamically constrained synthesis of the acoustic tomography, satellite altimetry, and in situ data (Lebedev et al. 2003), and in other studies, but it has not been used in the interpolation of the sparse SSC observations, which activates the present work.

Wang et al. (2018a) developed a three-dimensional sigma-coordinate suspended cohesive sediment transport model with the adjoint data assimilation, which has been used to investigate the parameter sensitivity (Wang et al. 2018a) and to estimate the parameters by assimilating in situ observations (Wang et al. 2017) or satellite observations (Wang et al. 2018b). Based on this model, a dynamically constrained interpolation methodology (DCIM) for the sparsely observed SSCs in both space and time is developed and applied in the Bohai Sea in this study. The details of the rest of the paper are as follows: the sparsely observed SSCs in the Bohai Sea, the dynamical model, and the hydrodynamic conditions are described in section 2 ; the DCIM is developed in section 3; the feasibility and validity of the DCIM are calibrated by ideal twin experiments in section 4; the DCIM is implemented in practical experiments in section 5; and the discussions and conclusions can be found in sections 6 and 7 , respectively.

\section{Observations and model}

\section{a. Observations}

A large area survey in the Bohai Sea was conducted from 0525 UTC 5 August to 0210 UTC 17 August 2015. 
Ninety-three stations were surveyed and each station was covered once. At each station water samples were collected at the surface, middle, bottom, or a combination of all of them. The SSCs were extracted in the laboratory using the water samples with the processes described in Bi et al. (2010). A total of 128 effective SSC observations were obtained at all the stations, as shown in Fig. 1, which were sparse in both space and time. The detailed statistical information of the SSC observations are shown in Table 1. As there were no in situ observations, the wind and hydrodynamic conditions during the observation period will be presented later using the numerical simulated results.
TABLE 1. Detailed statistical information of the SSC observations.

\begin{tabular}{lrcc}
\hline & No. & Max value $\left(\mathrm{mg} \mathrm{L}^{-1}\right)$ & Min value $\left(\mathrm{mg} \mathrm{L}^{-1}\right)$ \\
\hline Surface layer & 73 & 58.13 & 2.13 \\
Middle layer & 8 & 20.43 & 4.23 \\
Bottom layer & 47 & 84.80 & 1.93 \\
\hline
\end{tabular}

\section{b. The dynamical model}

The governing equation, boundary conditions, and initial conditions for the suspended cohesive sediment transport are as follows (Wang et al. 2018a):

$$
\begin{aligned}
& \frac{\partial C}{\partial t}+u \frac{\partial C}{\partial x}+v \frac{\partial C}{\partial y}+w \frac{\partial C}{H \partial \sigma}=\frac{\partial}{\partial x} \\
&-w_{s} C-\frac{K_{V}}{H} \frac{\partial C}{\partial \sigma}=E-D, \quad \sigma=0, \\
&-w_{s} C-\frac{K_{V}}{H} \frac{\partial C}{\partial \sigma}=0, \quad \sigma=1, \\
&\left.C\right|_{\partial \Omega_{1}}=C_{\mathrm{obc}}, \\
& \partial C /\left.\partial \mathbf{n}\right|_{\partial \Omega_{2}}=0, \\
& \partial C /\left.\partial \mathbf{n}\right|_{\partial \Omega_{3}}=0 \\
&\left.C\right|_{t=0}=C_{0},
\end{aligned}
$$

where $C$ represents the SSC; $t$ is the time; $x$ and $y$ are the horizontal coordinates; $\sigma$ is the vertical coordinate ( 0 for bottom and 1 for surface); $H$ is the total water depth, including the undisturbed water depth and the sea surface elevation; $u, v$, and $w$ are the flow velocity components in the $x, y$, and $\sigma$ directions, respectively; $K_{H}$ and $K_{V}$ are the horizontal and vertical diffusivity coefficients, respectively; $w_{s}$ denotes the suspended sediment settling velocity; $\partial \Omega_{1}, \partial \Omega_{2}$, and $\partial \Omega_{3}$ are the inflow open boundary, outflow open boundary, and solid boundary, respectively; $\mathbf{n}$ is the vector normal to the boundaries; $C_{\mathrm{obc}}$ denotes the SSCs at the inflow open boundary; $C_{0}$ is the initial conditions; and $E$ and $D$ are the erosion rate and the deposition rate, respectively, which are calculated with the well-known Partheniades-Krone formulations (Partheniades 1965):
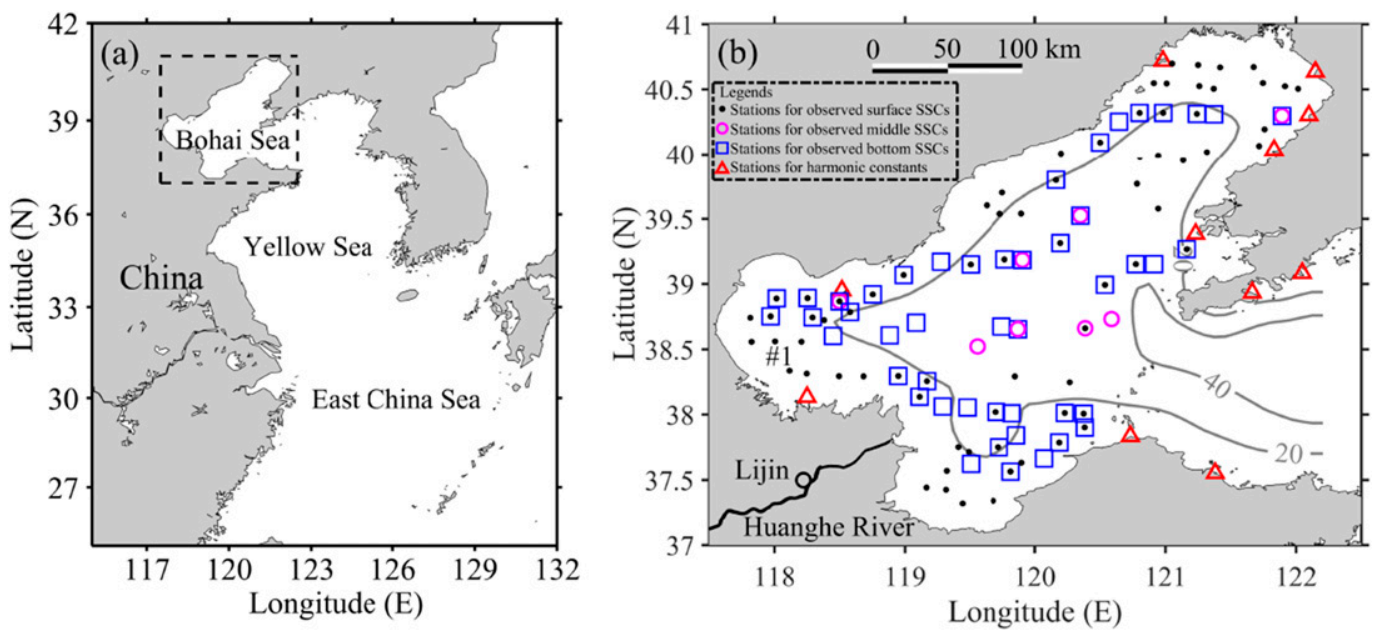

FIG. 1. (a) Map showing the general locations of the Bohai Sea (rectangle with dotted lines). (b) Map showing the locations of survey stations in the Bohai Sea, including the stations for observed surface SSCs (black dots), the stations for observed middle SSCs (magenta circles), the stations for observed bottom SSCs (blue squares), and the tide gauge stations (red triangles); the location of station 1; and bathymetric contours at 20 and $40 \mathrm{~m}$. 


$$
\begin{aligned}
& E= \begin{cases}M_{0}\left(\tau_{b} / \tau_{\mathrm{ce}}-1\right), & \tau_{b}>\tau_{\mathrm{ce}} \\
0, & \tau_{b} \leq \tau_{\mathrm{ce}}\end{cases} \\
& D=\left\{\begin{array}{ll}
w_{s} C_{1}\left(1-\tau_{b} / \tau_{\mathrm{cd}}\right), & \tau_{b}<\tau_{\mathrm{cd}} \\
0, & \tau_{b} \geq \tau_{\mathrm{cd}}
\end{array},\right.
\end{aligned}
$$

where $M_{0}$ is the resuspension rate; $\tau_{b}$ is the bottom shear stress; $\tau_{\mathrm{ce}}$ and $\tau_{\mathrm{cd}}$ are the critical shear stress for erosion and deposition, respectively; and $C_{1}$ is the SSC near the bottom.

Equations (1)-(9) are referred to as the dynamical model of the suspended cohesive sediment transport (i.e., the suspended cohesive sediment transport model), which will be used to constrain the gridded four-dimensional (both three-dimensional spatial and one-dimensional temporal) SSCs representing the interpolated results in this study. The numerical schemes for solving this dynamical model can be found in Wang et al. (2018a).

\section{c. Default model settings of the dynamical model}

The study area was the Bohai Sea, which was shown in Fig. 1. The horizontal resolution was $5 / 60^{\circ} \times 5 / 60^{\circ}$, and there were five uniform sigma layers in the vertical direction. The model was launched at 0525 UTC 5 August 2015. The integral time step was $300 \mathrm{~s}$, and the total simulation time was $284.75 \mathrm{~h}$ to cover the observation period.
The simulated results of the sediment transport are sensitive to the initial conditions (Wang et al. 2018a); therefore, the accurate specification of the initial values of SSCs is important for the sediment transport simulation over a short period (Lee et al. 2007; Yang et al. 2016). In this study the initial conditions were obtained by interpolating the observed SSCs. In the horizontal direction, the surface initial conditions were given by interpolating all the surface SSC observations using the surface spline interpolation (Guo et al. 2017) without regard to the time inconsistency, as shown in Fig. 2. Based on the turbidity observations in the Bohai Sea, the Yellow Sea, and the East China Sea, Bian et al. (2013) found that the surface layer SSC was much lower than the bottom layer SSC. Therefore, it is assumed that the average value of SSCs at every vertical layer was linearly decreased with the increasing vertical number of the layers ( 1 for the bottom layer and 5 for the surface layer). There were 29 stations where both the surface SSCs and the bottom SSCs were observed, and the average values of the surface SSCs and bottom SSCs at these stations were 9.50 and $13.63 \mathrm{mg} \mathrm{L}^{-1}$, respectively, with a standard deviation of 9.29 and $12.21 \mathrm{mg} \mathrm{L}^{-1}$, respectively. Therefore, the linear relationship between SSCs and the number of vertical layers $(k)$ was as follows: $C=14.67-1.03 \times k$. According to this linear formula, the initial conditions at the other layers were

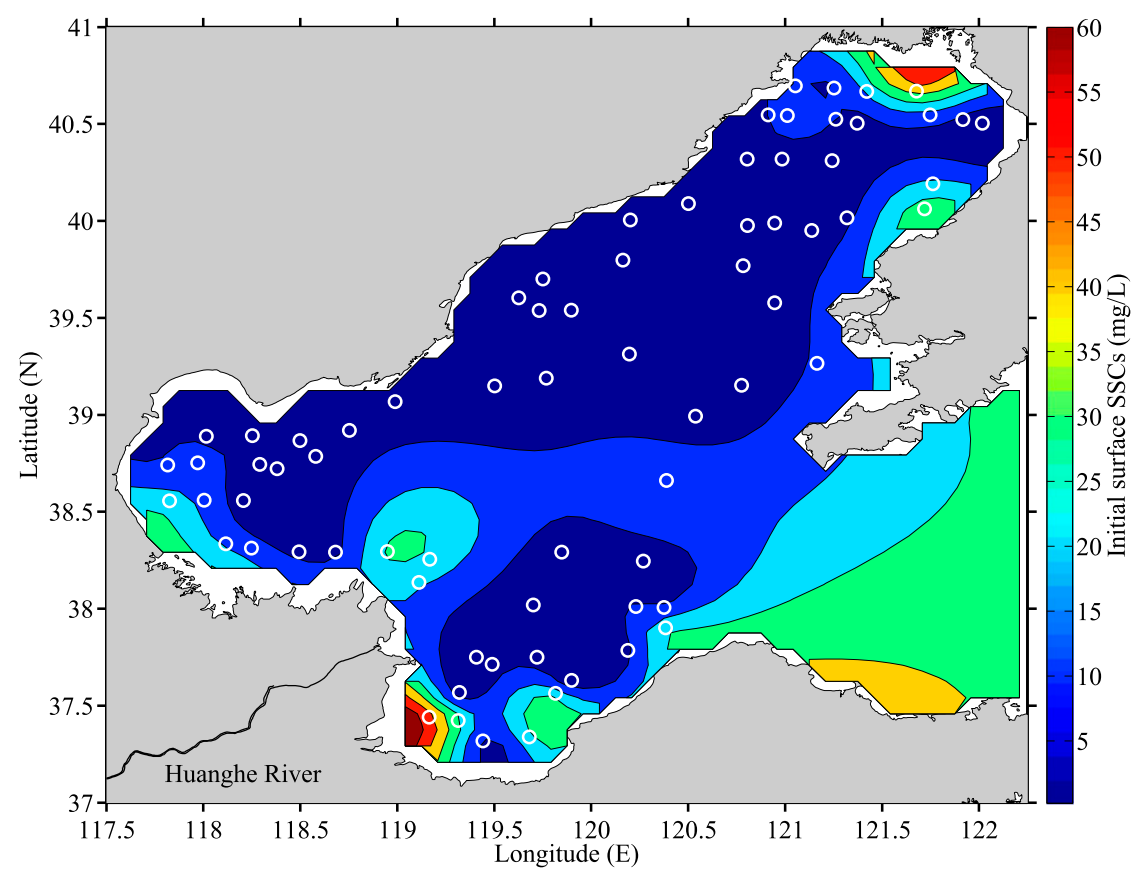

FIG. 2. Surface SSCs interpolated using the surface observations without regard to the time inconsistency (colors) and locations of the observations at the surface layer (white circles). 

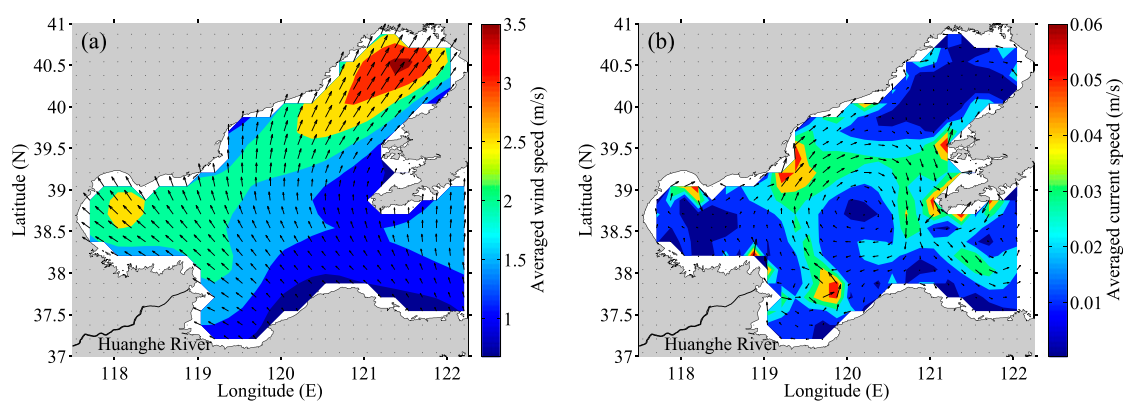

FIG. 3. (a) The temporally averaged sea surface wind speeds (colors) and directions (arrows). (b) The vertically and temporally averaged current speeds (colors) and directions (arrows). It was noted that the sea surface wind and current were temporally averaged during the observation period from 0525 UTC 5 Aug to 0210 UTC 17 Aug 2015.

given by multiplying the surface initial conditions with coefficients $(1.5436-0.1087 \times k)$.

The monthly averaged SSCs in August 2015 at the Lijin station was about $858.2 \mathrm{mg} \mathrm{L}^{-1}$ according to YRCCMWRC (2015). As the Lijin station was about $100 \mathrm{~km}$ upstream of the mouth of the Huanghe River, the inflow open boundary forcing at the mouth of the Huanghe River was specified by multiplying the SSCs at the Lijin station by a factor of 0.1 according to Jiang et al. (2004). The settling velocity and resuspension rate were set to $1.00 \times 10^{-4} \mathrm{~m} \mathrm{~s}^{-1}$ and $5.00 \times 10^{-6} \mathrm{~kg} \mathrm{~m}^{-2} \mathrm{~s}^{-1}$, respectively. The critical erosion stress and the critical deposition stress were equal in the present model, which were set to $0.36 \mathrm{~N} \mathrm{~m}^{-2}$ according to Zuo et al. (2014).

\section{d. The hydrodynamic conditions}

The hydrodynamic background field was calculated using ROMS (Song and Haidvogel 1994). The spatial and temporal resolutions were the same as those in the suspended sediment transport model. The monthaverage runoffs of the Huanghe River were given as the upstream boundaries according to YRCCMWRC (2015). The hourly winds of the NCEP Climate Forecast System Reanalysis (Saha et al. 2014) were taken as the surface wind forcing. The wind forcing was temporally averaged during the observation period from 0525 UTC 5 August to 0210 UTC 17 August 2015, and the results were shown in Fig. 3a, indicating that the winds were mainly from sea to land and that the average wind
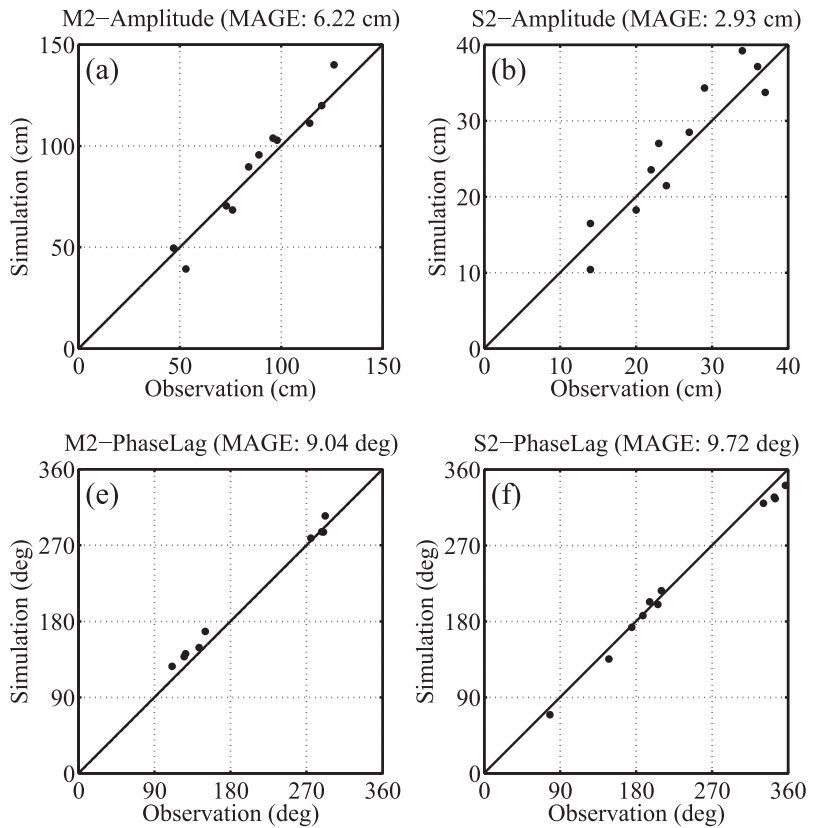
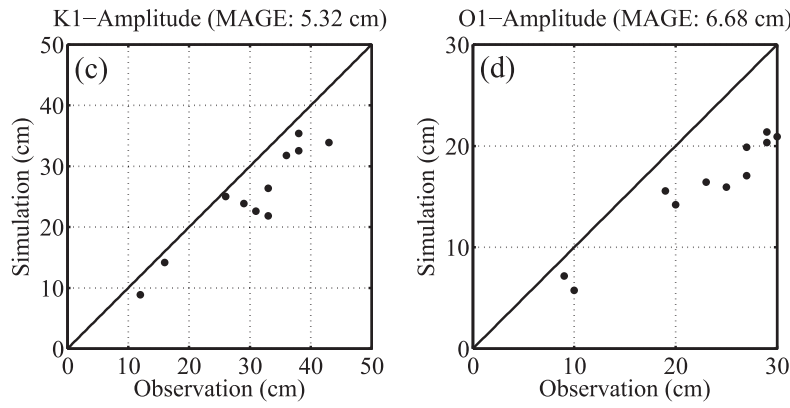

K1-PhaseLag (MAGE: $4.79 \mathrm{deg})$
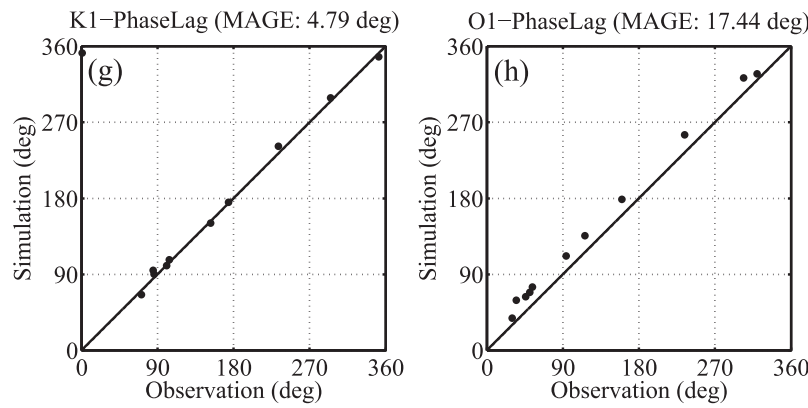

FIG. 4. Comparison of simulated and observed amplitude for (a) $\mathrm{M}_{2}$, (b) $\mathrm{S}_{2}$, (c) $\mathrm{K}_{1}$, and (d) $\mathrm{O}_{1}$. Comparison of simulated and observed phase lag for (e) $\mathrm{M}_{2}$, (f) $\mathrm{S}_{2}$, (g) $\mathrm{K}_{1}$, and (h) $\mathrm{O}_{1}$. The 1:1 lines are shown for reference in all the panels. 
speed in the Bohai Sea was about $1.93 \mathrm{~m} \mathrm{~s}^{-1}$. At the seaward open boundaries, four dominating constituents were considered, including $\mathrm{M}_{2}, \mathrm{~S}_{2}, \mathrm{~K}_{1}$, and $\mathrm{O}_{1}$. The harmonic constants were obtained from Oregon State University Tidal Inversion Software (Egbert and Erofeeva 2002).

The simulated current velocities were first averaged vertically and then averaged temporally during the observation period from 0525 UTC 5 August to 0210 UTC 17 August 2015, and the results were shown in Fig. 3b. The main pattern showed that there was an anticyclonic circulation in the middle of the Bohai Sea that was consistent with the simulated results during summertime in Liu et al. (2003) and Wan et al. (2004). The harmonic constants of each constituent, analyzed from the simulated water levels of 30 days, were compared with the observed data at 11 tide gauge stations (see Fig. 1), which were shown in Fig. 4. The minimum value of the amplitudes of the $\mathrm{M}_{2}$ tide was larger than the maximum values of the other three constituents (see Figs. $4 \mathrm{a}-\mathrm{d}$ ), showing that the $\mathrm{M}_{2}$ constituent dominated the tidal forcing, which was consistent with the results in Fang et al. (2004). The mean absolute gross error (MAGE) was used to quantify the discrepancy between the simulated results and the observations, which was calculated as follows (Zhang et al. 2012):

$$
\text { MAGE }=\frac{1}{N} \sum_{i=1}^{N}\left|M_{i}-O_{i}\right|
$$

where $N$ was the number of observations; and $M$ and $O$ were the modeled and observed results, respectively. For all the constituents, the MAGEs between the simulated and observed amplitude were less than $7 \mathrm{~cm}$, while the MAGEs for the phase lag were less than $18^{\circ}$, indicating that the simulated hydrodynamic background field was acceptable for driving the suspended sediment transport model for this study.

\section{Dynamically constrained interpolation methodology}

The DCIM combines dynamical constraints from the numerical model of dynamical processes with statistical information from observations, so it can produce dynamically and statistically consistent estimates of the entire study area and naturally blend several types of observations (Yaremchuk and Sentchev 2013). In the DCIM the dynamic numerical model, which provides the dynamical constrains, is used to interpolate the observations; in addition, the interpolated results are optimized iteratively by adjusting the key model parameters using the adjoint method (Sasaki 1970; Thacker and Long 1988; Navon 1998).

\section{a. The adjoint method}

When the adjoint method is used, a cost function is first defined to measure the squared differences between the interpolated results and the observations, as follows:

$$
J=\frac{1}{2} \sum_{i, j, k, n} W_{i, j, k}^{n}\left(C_{i, j, k}^{n}-\tilde{C}_{i, j, k}^{n}\right)^{2}
$$

where $C_{i, j, k}^{n}$ and $\tilde{C}_{i, j, k}^{n}$ are the interpolated and observed SSCs at the $(i, j, k)$ grid point at the $n$th time step, respectively; $\mathbf{W}$ is the weighting matrix and theoretically should be the inverse of the observation error covariance matrix, which can be simplified by assuming that the errors of the data are uncorrelated and equally weighted (Yu and O'Brien 1992). In the present study, the elements in $\mathbf{W}$ are 1 where observations are available and 0 otherwise.

According to the theory of the Lagrangian multiplier method, the Lagrangian function is defined as

$$
L=J+\sum_{i, j, n}\left\{\begin{array}{l}
\sum_{k=1}^{K}\left\{\lambda_{i, j, k}^{n} \times \text { discretization of [left side of Eq. (1) - right side of Eq. (1)] }\right\} \\
+\frac{\lambda_{i, j, 0}^{n}+\lambda_{i, j, 1}^{n}}{2} \times \text { discretization of [right side of Eq. (2) - left side of Eq. (2)] } \\
+\frac{\lambda_{i, j, K}^{n}+\lambda_{i, j, K+1}^{n}}{2} \times \text { discretization of [right side of Eq. (3) - left side of Eq. (3)] }
\end{array}\right\}
$$

where $\lambda$ denotes the adjoint variable of $C$; and $K$ is the total vertical layers for the water column, and there is one false layer at the bottom, numbered 0 , while $K+1$ represents the false layer at the surface.
To minimize the cost function, the first-order derivate of the Lagrangian function with respect to the variables and parameters should be zero (Thacker and Long 1988): 


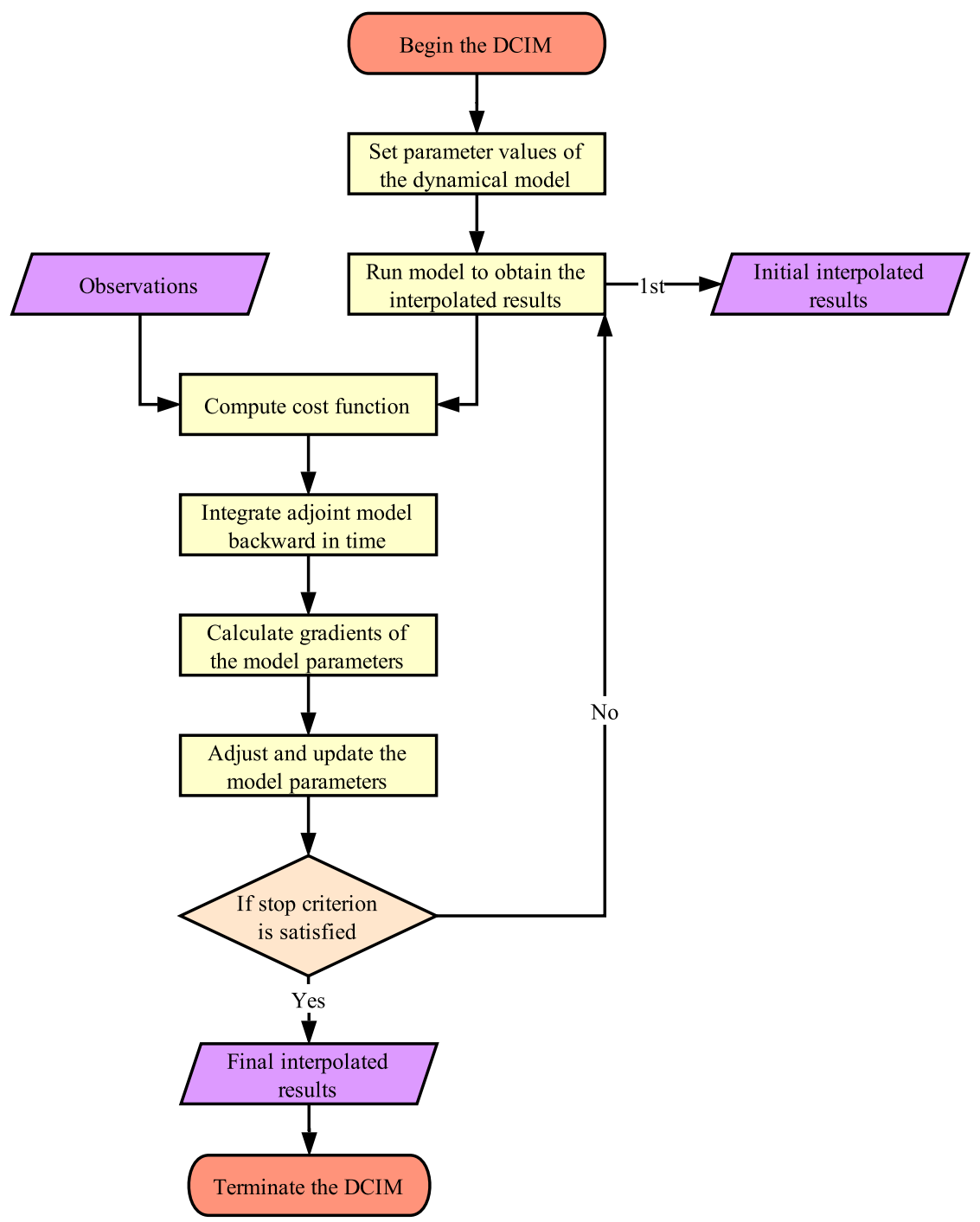

FIG. 5. Flowchart for the implementation of the DCIM.

$$
\begin{gathered}
\frac{\partial L}{\partial \lambda_{i, j, k}^{n}}=0, \\
\frac{\partial L}{\partial C_{i, j, k}^{n}}=0, \\
\frac{\partial L}{\partial p_{i, j, k}^{n}}=0,
\end{gathered}
$$

where $p$ denotes the parameters in the suspended cohesive sediment transport model, including $K_{H}, K_{V}, w_{s}$, $M_{0}, C_{\mathrm{obc}}, \tau_{\mathrm{ce}}, \tau_{\mathrm{cd}}$, and $C_{0}$. In fact, Eq. (13) returns the discretization of Eq. (1). From Eq. (14) the discrete adjoint model can be derived, which governs the evolution of the adjoint variable $\lambda$. From Eq. (15) gradients of the cost function with respect to model parameters are obtained. The detailed formulas of the adjoint model and the gradients can be found in Wang et al. (2018a).

\section{b. The process of DCIM}

As a general overview, the DCIM involves the following steps:

Step 1. Set the initial guess values of all the model parameters in the dynamical numerical model [i.e., Eqs. (1)-(9) in this study].

Step 2. Run the dynamic numerical model to obtain the interpolated results.

Step 3. Compute the cost function, as shown in Eq. (11).

Step 4. Integrate the adjoint model, derived from Eq. (14), backward in time to get the adjoint variables. 
Step 5. Calculate the gradients of the cost function with respect to the key model parameters, which are derived from Eq. (15).

Step 6. Adjust the key model parameters using the steepest descent method and update the values of the key model parameters in the dynamical numerical model.

Step 7. Check whether the cost function has satisfied the requirement of minimization. If the stop criterion is satisfied, then terminate the iteration; otherwise, return to step 2 .

The corresponding flowchart is shown in Fig. 5. Considering the number of sparse observations is small, the adjusted key model parameters, except for the initial conditions, are assumed to be constant to prevent the ill-posedness of the inverse problem; in addition, all the key model parameters are adjusted synchronously.

In this study the sparsely observed SSCs were interpolated in space and time following the aforementioned processes. When the DCIM was implemented, the key model parameters, including spatially varying initial conditions, constant settling velocity, constant critical shear stresses for deposition and erosion, and constant resuspension rate, were adjusted synchronously, while the other model parameters were not changed. The stop criterion in step 7 was that the difference of the cost functions, normalized by the values at the first iteration step, between the last two iteration steps was less than $1.00 \times 10^{-6}$, with a maximum value of 100 for iteration steps in the twin experiments and 500 for the practical experiments.

\section{Verification of the dynamically constrained interpolation methodology}

In this section the feasibility and validity of the DCIM were verified by ideal twin experiments, in which only the spatiotemporal locations of the practical observations were used. The SSC observations were generated artificially by running the suspended sediment transport model with the default model settings. The constant model parameters, including settling velocity, resuspension rate, and critical shear stresses, were estimated synchronously in the twin experiments, while the initial conditions, which varied spatially, were not estimated. Without the loss of generality, the initial guess values of the estimated model parameters were set to be half of the default values. The similar conclusions were obtained when the other initial guess values were set, which were not shown in the present study for brevity.

\section{a. Application of DCIM in twin experiments}

As mentioned by Elbern et al. (2007), the validity of the assimilated or interpolated results can only be shown satisfactorily by independent observations that were not assimilated or interpolated. Therefore, one-eighth of the total observations were randomly selected as independent observations, which were not interpolated but used only for verification and were named as checked observations. The other observations were labeled as interpolated observations, which were interpolated using the DCIM.

To eliminate the contingency introduced by the selection of checked observations, all the artificial observations were randomly split into eight subsets and every subset was taken as the checked observations in a twin experiment. Therefore, there were eight twin

TABLE 2. Statistics of the twin experiments at the initial and final iteration steps of DCIM.

\begin{tabular}{|c|c|c|c|c|c|c|c|c|}
\hline \multirow[b]{2}{*}{ Expt } & \multicolumn{2}{|c|}{$\mathrm{K} 1^{\mathrm{a}}$} & \multicolumn{2}{|c|}{$\mathrm{K} 2^{\mathrm{b}}$} & \multicolumn{2}{|c|}{$\mathrm{K} 3^{\mathrm{c}}$} & \multicolumn{2}{|c|}{$\mathrm{K} 4^{\mathrm{d}}$} \\
\hline & Initial & Final & Initial (\%) & Final (\%) & Initial & Final & Initial (\%) & Final (\%) \\
\hline TE_11 & 26.81 & 0.20 & 341.96 & 3.73 & 38.34 & 0.32 & 340.21 & 3.23 \\
\hline TE_12 & 28.56 & 0.17 & 345.48 & 3.41 & 26.09 & 0.15 & 315.62 & 3.18 \\
\hline TE_13 & 26.95 & 0.31 & 348.80 & 5.14 & 37.34 & 0.44 & 292.33 & 4.37 \\
\hline TE_14 & 28.37 & 0.17 & 343.29 & 3.12 & 27.43 & 0.13 & 330.90 & 3.62 \\
\hline TE_15 & 28.33 & 0.12 & 331.35 & 2.09 & 27.68 & 0.14 & 414.47 & 2.76 \\
\hline TE_16 & 28.21 & 0.16 & 347.32 & 2.86 & 28.56 & 0.14 & 302.72 & 2.59 \\
\hline TE_17 & 29.49 & 0.24 & 341.45 & 3.95 & 19.56 & 0.17 & 343.83 & 4.39 \\
\hline TE_18 & 29.28 & 0.14 & 334.30 & 2.64 & 21.03 & 0.16 & 393.88 & 2.43 \\
\hline TE_21 & 28.25 & 0.18 & 341.74 & 3.27 & - & - & - & - \\
\hline TE_31 & 28.25 & 0.26 & 341.74 & 2.53 & - & - & - & - \\
\hline TE_32 & 28.25 & 0.36 & 341.74 & 4.25 & - & - & - & - \\
\hline TE_33 & 28.25 & 0.74 & 341.74 & 10.78 & - & - & - & - \\
\hline
\end{tabular}

${ }^{a} \mathrm{~K} 1$ is MAGEs between the interpolated observations and the corresponding interpolated SSCs (mg $\mathrm{L}^{-1}$ ).

${ }^{\mathrm{b}} \mathrm{K} 2$ is MNGEs between the interpolated observations and the corresponding interpolated SSCs.

${ }^{\mathrm{c}} \mathrm{K} 3$ is MAGEs between the checked observations and the corresponding interpolated SSCs (mg L ${ }^{-1}$ ).

${ }^{\mathrm{d}} \mathrm{K} 4$ is MNGEs between the checked observations and the corresponding interpolated SSCs. 

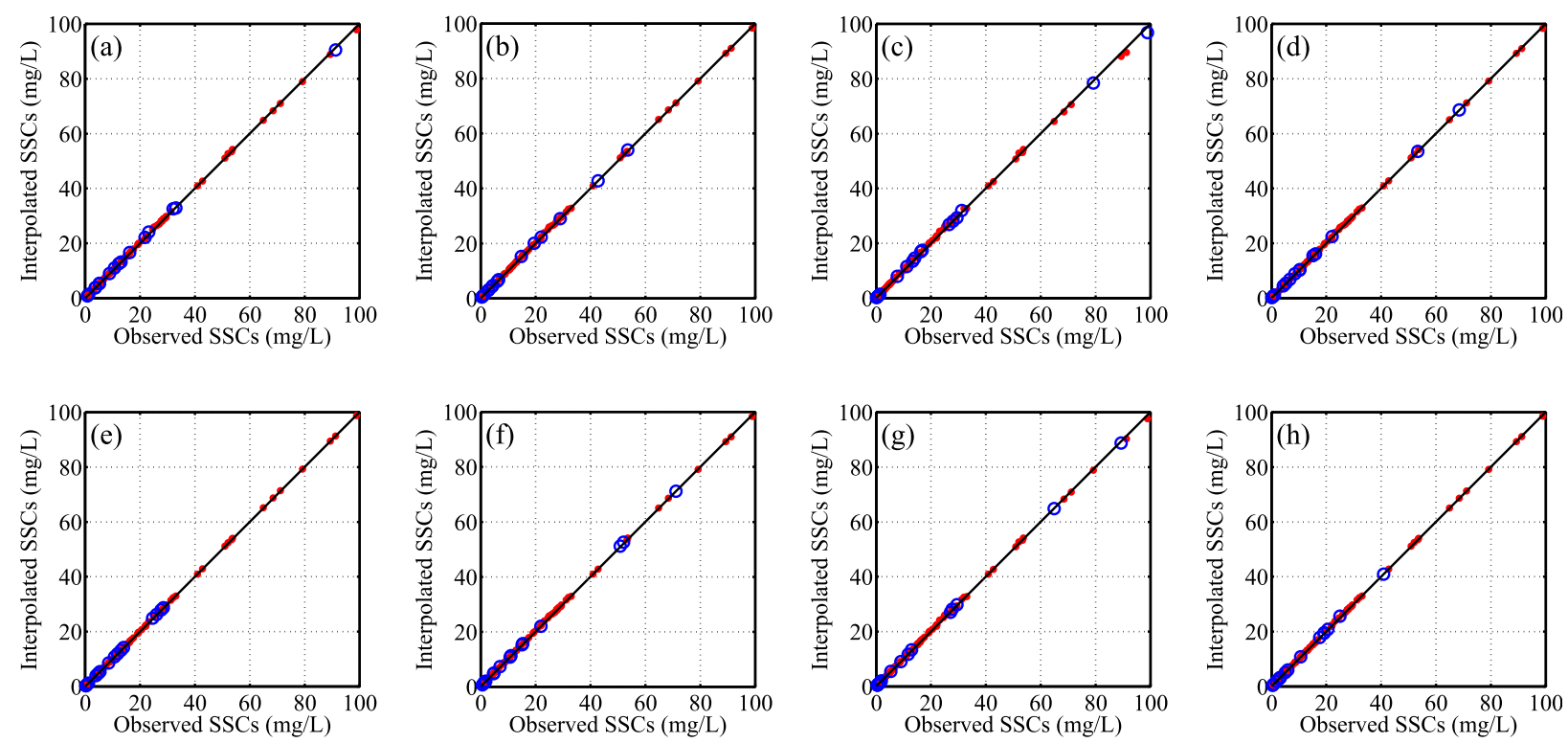

FIG. 6. Comparison of simulated and observed SSCs, including interpolated observations (red dots) and checked observations (blue circles), for (a) TE_11, (b) TE_12, (c) TE_13, (d) TE_14, (e) TE_15, (f) TE_16, (g) TE_17, and (h) TE_18. The 1:1 lines are shown for reference in all the panels.

experiments, which were named as TE_11-TE_18. The statistics of these twin experiments are listed in Table 2. At the initial iteration step, the MAGEs between the checked observations and the corresponding interpolated SSCs were on the order of magnitude of $10^{1} \mathrm{mg} \mathrm{L}^{-1}$. It should be noted that the averaged value of all the artificial observations was also on the order of magnitude of $10^{1} \mathrm{mg} \mathrm{L}^{-1}$, which was comparable with the magnitude of MAGEs. Therefore, the mean normalized gross error (MNGE) was used to further quantify the discrepancy between the interpolated SSCs and observations, which was calculated as follows (Zhang et al. 2012):

$$
\mathrm{MNGE}=\frac{1}{N} \sum_{i=1}^{N}\left[\left(\left|I_{i}-O_{i}\right|\right) / O_{i}\right]
$$

where $N$ was the number of observations; and $I$ and $O$ were the interpolated SSCs and observations, respectively. The calculated results are also listed in Table 2. All the MNGEs between the checked observations and the corresponding interpolated SSCs at the initial iteration step were larger than $290 \%$, which were similar to those between the interpolated observations and the corresponding interpolated SSCs. The large discrepancies were introduced by the difference between the initial guess values and the true values of the adjusted model parameters, which indicated that the suspended sediment transport model was sensitive to the adjusted model parameters, which was the same as that in Wang et al. (2018a). When the DCIM was implemented, the MAGEs between the checked observations and the corresponding interpolated SSCs were reduced by an order of $10^{2}$, which were the same as those between the interpolated observations and the corresponding interpolated SSCs; in addition, all the MNGEs between the interpolated observations and the interpolated SSCs were less than 6\%, while all the MNGEs between the checked observations and the interpolated SSCs were less than 5\%. The scatterplots in Fig. 6 indicated that the correlation coefficient between the final interpolated SSCs and the artificial observations (both checked observations and interpolated observations) was nearly 1.00 in every twin experiment, showing the DCIM was an effective method to interpolate the sparse artificial SSC observations.

To obtain the optimal interpolation results, all the artificial observations were interpolated in a new twin experiment named TE_21. The MAGE between the final interpolated SSCs and the observations was $0.18 \mathrm{mg} \mathrm{L}^{-1}$, while the MNGE was $3.27 \%$ (see Table 2). In addition, the correlation coefficient between the final interpolated SSCs and the artificial observations in TE_21 was 1.00, as shown in Fig. 7a. It indicated that the final interpolated SSCs in TE_21 were almost equal to the artificial SSC observations. During the iteration processes in TE_21, the normalized cost function and the $L_{2}$ norm of gradients of the cost function with respect to the adjusted model parameters, including settling velocity, resuspension 

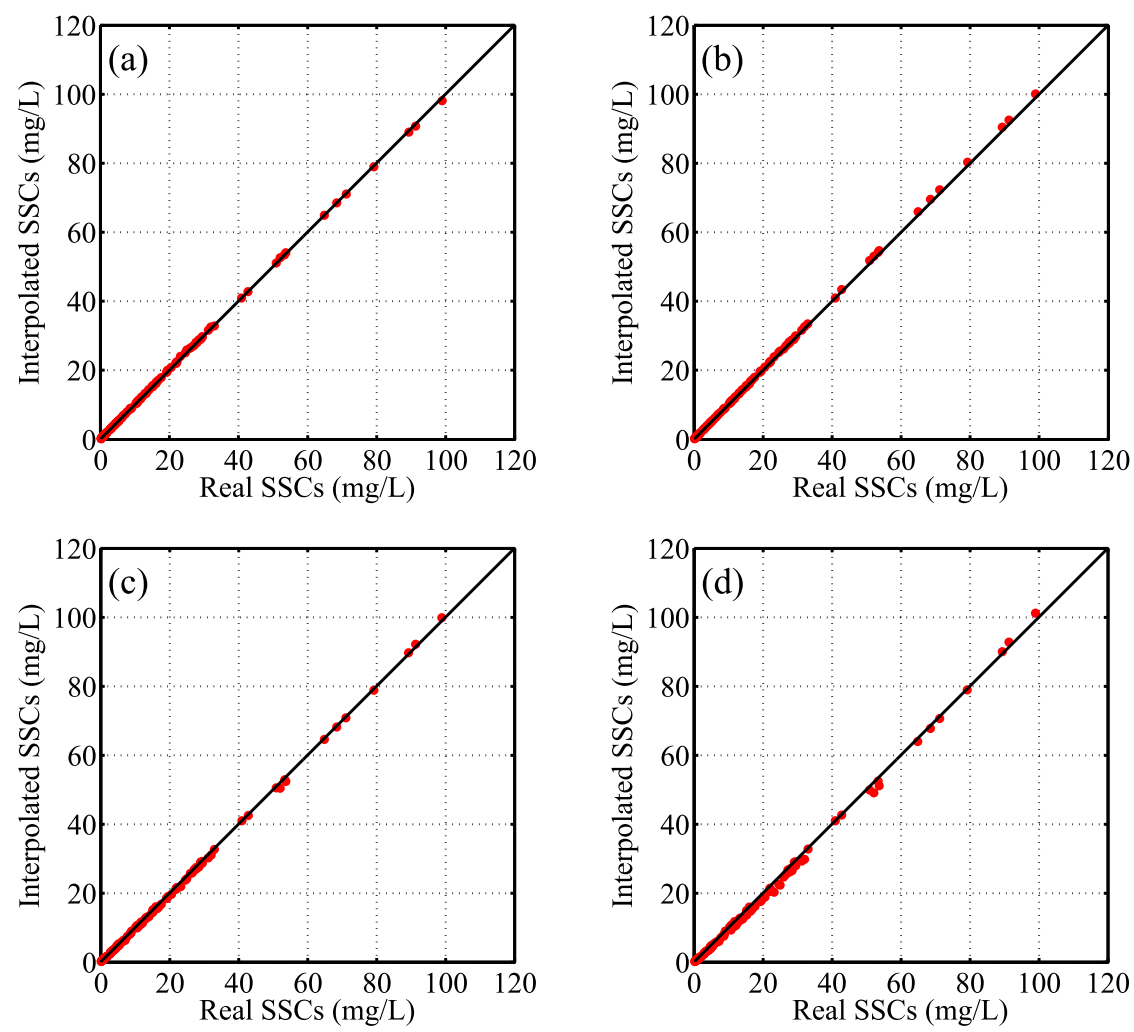

FIG. 7. Comparison of simulated and true values of SSCs for (a) TE_21, (b) TE_31, (c) TE_32, and (d) TE_33. The 1:1 lines are shown for reference in all the panels.

rate, and critical shear stresses, were reduced stably and largely (see Fig. 8), showing that the adjustment of these model parameters was reasonable and effective. The modeling results indicated that through iteratively adjusting the key model parameters, the DCIM can effectively improve the interpolation results and that the final interpolated SSCs were close to the artificial observations, demonstrating that the DCIM was a feasible and effective method for interpolating the sparsely observed SSCs.

\section{b. Sensitivity to observational errors}

In reality the oceanic observed SSCs are not perfect and usually contain noises. Therefore, three new twin experiments, including TE_31, TE_32, and TE_33, were carried out, in which the artificial observations were contaminated by random perturbations. The maximum percentage errors were $10 \%, 20 \%$, and $30 \%$ in TE_31, TE_32, and TE_33, respectively. It was noted that the contaminated observations were interpolated and that the true values of SSCs were used in the calculations of MAGEs and MNGEs. When the DCIM was used, all the final interpolated SSCs in these three twin experiments were close to the true values of SSCs, as shown in Figs. 7b-d. As the maximum percentage error became larger, the MAGEs, the normalized cost function, and the $L_{2}$ norm of gradients of the cost function with respect to the estimated parameters were increased (see Table 2; Fig. 8); however, the MNGE was the minimum in TE_31 (see Table 2). It showed that although the mean distance between the interpolated SSCs and the true values of SSCs in TE_31 was larger than that in TE_21, the interpolated SSCs were much close to the true values of SSCs overall, indicating that the small observational errors can be ignored. The maximum percentage error was $30 \%$ in TE_33, but the MNGE between the final interpolated results and the true values of SSCs was just $10.78 \%$. From the results of TE_31-TE_33, it can be concluded that the DCIM was applicable and effective when the SSC observations were contaminated by data noise, which indicated the potential to be applied in practical applications.

\section{Practical applications}

In practical experiments, the practical sparsely observed SSCs were interpolated using the DCIM. The initial guess values of all the model parameters in the dynamical model were set to the default values. In addition, the model parameters, including spatially varying initial conditions, 

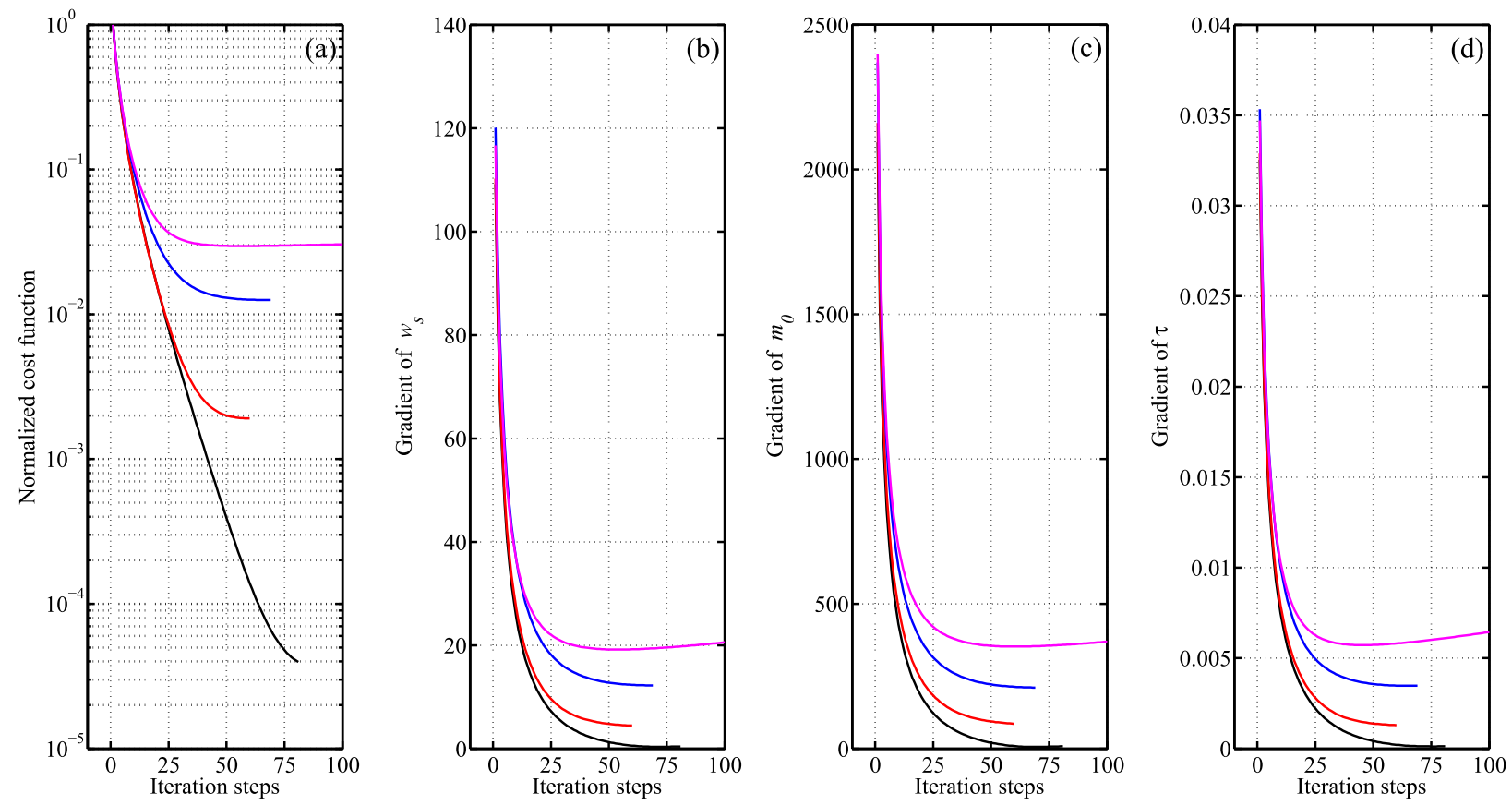

FIG. 8. Variations of (a) normalized cost function, and $L_{2}$ norm of gradients of the cost function with respect to (b) settling velocity, (c) resuspension rate, and (d) critical stress in TE_21 (black lines), TE_31 (red lines), TE_32 (blue lines), and TE_33 (magenta lines).

constant settling velocity, constant critical shear stresses for deposition and erosion, and constant resuspension rate, were adjusted synchronously.

\section{a. Validation of DCIM in practical experiments}

To test the validity of the DCIM in practical applications, all the practical SSC observations were
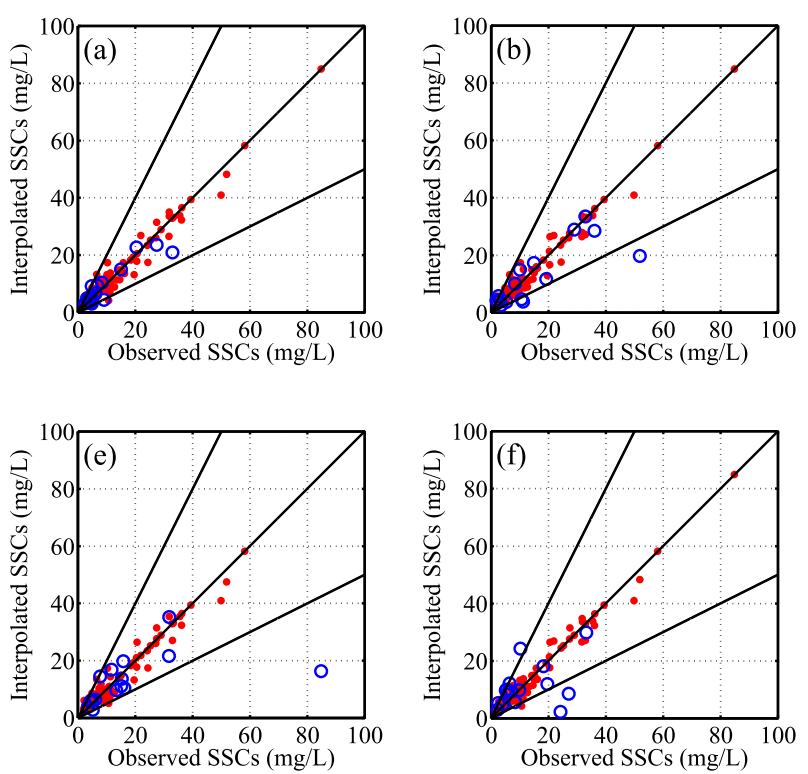

randomly split into eight subsets and every subset was taken as the checked observations in a practical experiment. Therefore, there were eight practical experiments, which were named as PE_11-PE_18. As shown in Fig. 9, the interpolated results captured a majority of interpolated observations with a factor of 2 , whose minimum value was $96.43 \%$, which occurred
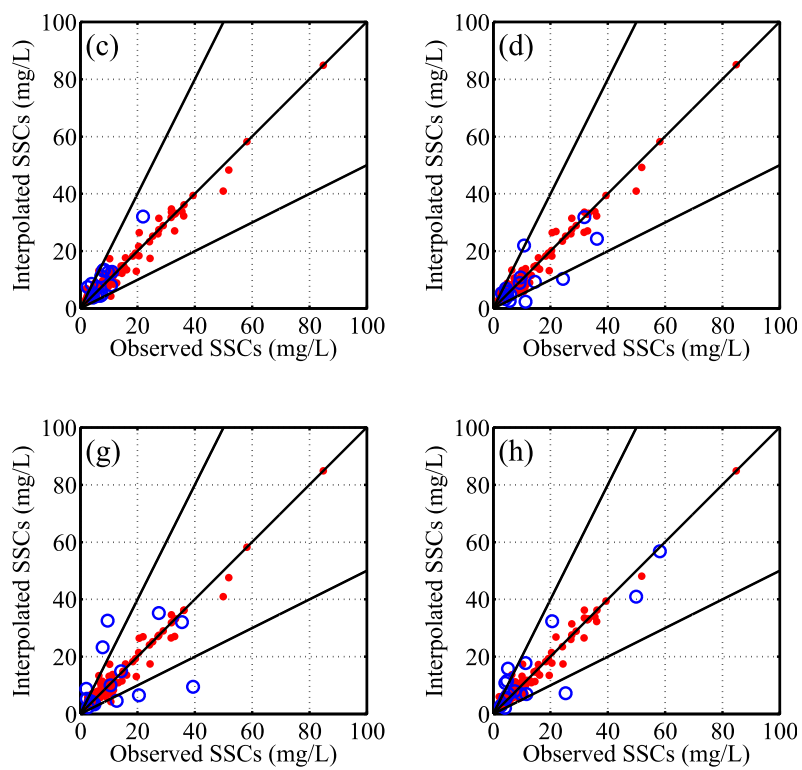

FIG. 9. Comparison of simulated and observed SSCs, including interpolated observations (red dots) and checked observations (blue circles), for (a) PE_11, (b) PE_12, (c) PE_13, (d) PE_14, (e) PE_15, (f) PE_16, (g) PE_17, and (h) PE_18. The 1:2, 1:1, and 2:1 lines are shown for reference in all the panels. 
TABLE 3. Statistics of the practical experiments at the initial and final iteration steps of DCIM.

\begin{tabular}{|c|c|c|c|c|c|c|c|c|}
\hline \multirow[b]{2}{*}{ Expt } & \multicolumn{2}{|c|}{$\mathrm{K} 1^{\mathrm{a}}$} & \multicolumn{2}{|c|}{$\mathrm{K} 2^{\mathrm{b}}$} & \multicolumn{2}{|c|}{$\mathrm{K} 3^{\mathrm{c}}$} & \multicolumn{2}{|c|}{$\mathrm{K} 4^{\mathrm{d}}$} \\
\hline & Initial & Final & Initial (\%) & Final (\%) & Initial & Final & Initial (\%) & Final (\%) \\
\hline PE_11 & 13.01 & 1.54 & 168.15 & 19.16 & 115.81 & 2.45 & 156.11 & 28.82 \\
\hline PE_12 & 13.35 & 1.41 & 176.50 & 17.09 & 9.21 & 5.06 & 97.66 & 44.71 \\
\hline PE_13 & 11.99 & 1.46 & 149.65 & 18.46 & 18.73 & 3.26 & 285.59 & 48.75 \\
\hline PE_14 & 12.78 & 1.40 & 165.91 & 17.60 & 13.18 & 4.14 & 171.78 & 38.68 \\
\hline PE_15 & 13.40 & 1.46 & 183.40 & 19.49 & 8.89 & 7.51 & 49.38 & 33.37 \\
\hline PE_16 & 12.62 & 1.39 & 161.83 & 17.30 & 14.33 & 5.82 & 200.32 & 55.06 \\
\hline PE_17 & 12.12 & 1.46 & 150.74 & 17.64 & 17.81 & 7.27 & 277.99 & 76.65 \\
\hline PE_18 & 13.39 & 1.32 & 176.98 & 17.58 & 8.94 & 5.27 & 94.32 & 57.05 \\
\hline PE_21 & 12.83 & 1.54 & 166.64 & 19.32 & - & - & - & - \\
\hline
\end{tabular}

${ }^{\mathrm{a}} \mathrm{K} 1$ is MAGEs between the interpolated observations and the corresponding interpolated SSCs $\left(\mathrm{mg} \mathrm{L}^{-1}\right)$.

${ }^{\mathrm{b}} \mathrm{K} 2$ is MNGEs between the interpolated observations and the corresponding interpolated SSCs.

${ }^{\mathrm{c}} \mathrm{K} 3$ is MAGEs between the checked observations and the corresponding interpolated SSCs $\left(\mathrm{mg} \mathrm{L}^{-1}\right)$.

${ }^{\mathrm{d}} \mathrm{K} 4$ is MNGEs between the checked observations and the corresponding interpolated SSCs.

in PE_15 (see Fig. 9e). Conversely, the checked observations were captured less and the minimum value was $56.25 \%$ in PE_17 (see Fig. 9g). The MAGEs between the interpolated observations and the corresponding interpolated SSCs were decreased by at least $87.81 \%$, and the corresponding MNGEs were decreased by $87.67 \%$, which occurred in PE_13 (see Table 3). The MAGEs and MNGEs between the final interpolated SSCs and the checked observations were slightly large, but they were all much less than those at the initial iteration step (see Table 3). As indicated above, restricted by the number of observations the adjusted model parameters in the DCIM had to be

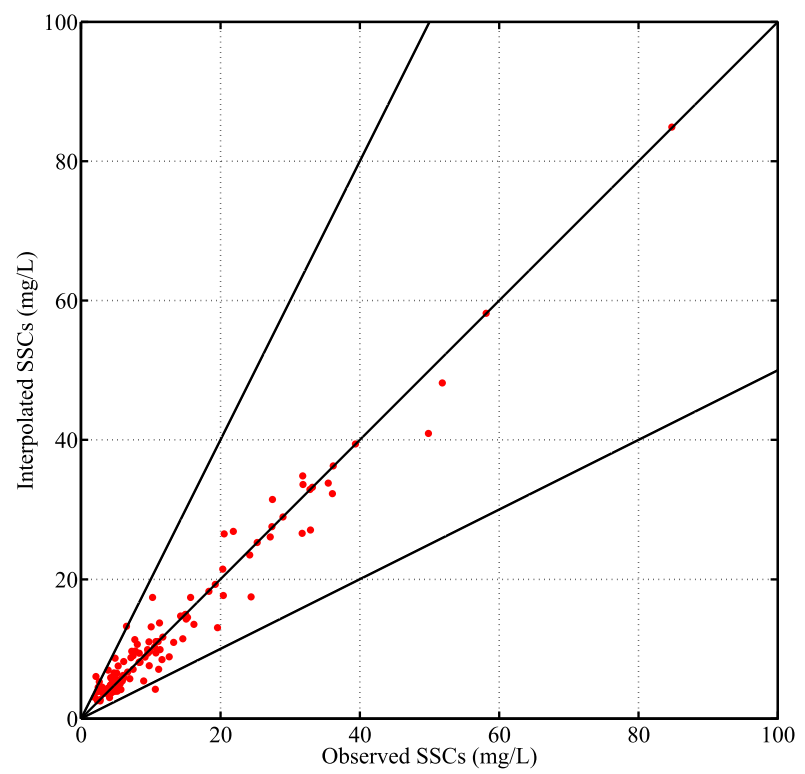

FIG. 10. Comparison of simulated and observed SSCs for PE_21. The 1:2,1:1, and 2:1 lines are shown for reference. assumed to be constant, which would introduce modeling errors (Wang et al. 2017). On the whole, the final interpolated SSCs agreed well with the interpolated observations, and the discrepancies between the final interpolated SSCs and the checked observations were in a reasonable range, demonstrating that the DCIM were also effective in the practical applications.

\section{b. Optimal interpolated results}

To obtain the optimal results, all the practical observations were interpolated in PE_21. The MAGE and MNGE between the final interpolated SSCs and the observed SSCs were $1.54 \mathrm{mg} \mathrm{L}^{-1}$ and $19.32 \%$ (see Table 3), respectively, which were reduced by an order of magnitude. The mean value and the standard deviation of all the SSC observations were 12.54 and $12.93 \mathrm{mg} \mathrm{L}^{-1}$, respectively, while those of the final interpolated SSCs were 12.57 and $12.47 \mathrm{mg} \mathrm{L}^{-1}$. The correlation coefficient between the SSC observations and the final interpolated SSCs was 0.98. Furthermore, the final interpolated results captured a majority $(96.88 \%)$ of observations with a factor of 2, as shown in Fig. 10. The aforementioned results indicated the interpolated SSCs in PE_21 agreed well with the practical observations. Besides, the normalized cost function and the $L_{2}$ norm of gradients of the cost function with respect to the adjusted model parameters were reduced rapidly and tended to be stable (see Fig. 11), showing that these model parameters were adjusted reasonably and that the interpolated results were fairly good.

The final interpolated surface SSC at station 1, whose location was shown in Fig. 1, was equal to the observed surface SSC (see Fig. 12a). In addition, the time series of the interpolated surface SSCs and the height above the seabed at station 1 presented 

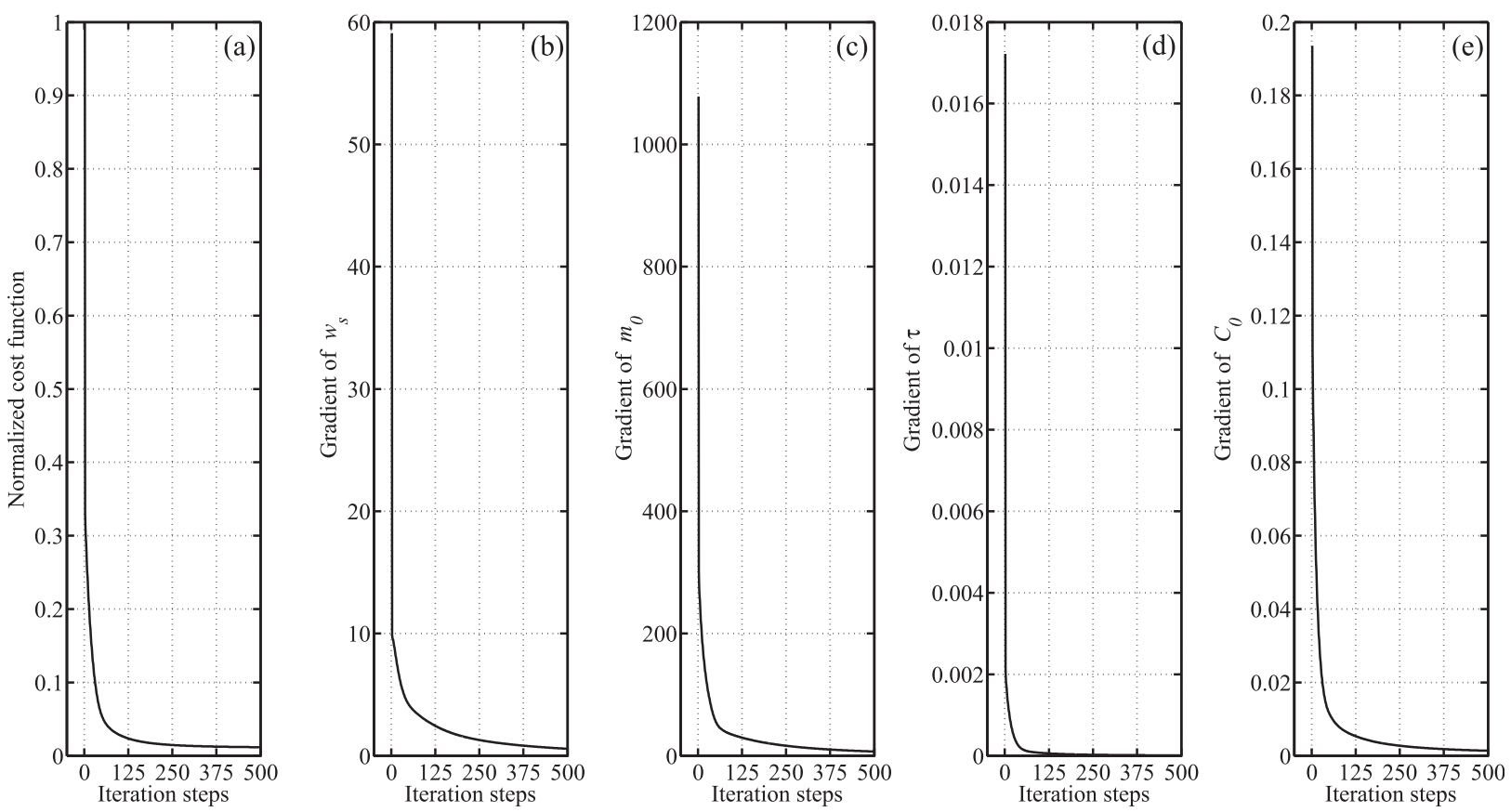

FIG. 11. Variations of (a) normalized cost function, and $L_{2}$ norm of gradients of the cost function with respect to (b) settling velocity,

(c) resuspension rate, (d) critical stress, and (e) initial conditions in PE_21.

clear periodic variations (see Fig. 12a), in which the semidiurnal variation was the strongest followed by the diurnal variation (see Figs. 12b and 12c). Besides, the temporal variations of the interpolated surface SSCs and the tidal elevation at station 1 had a significantly negative correlation (see Fig. 12a), which
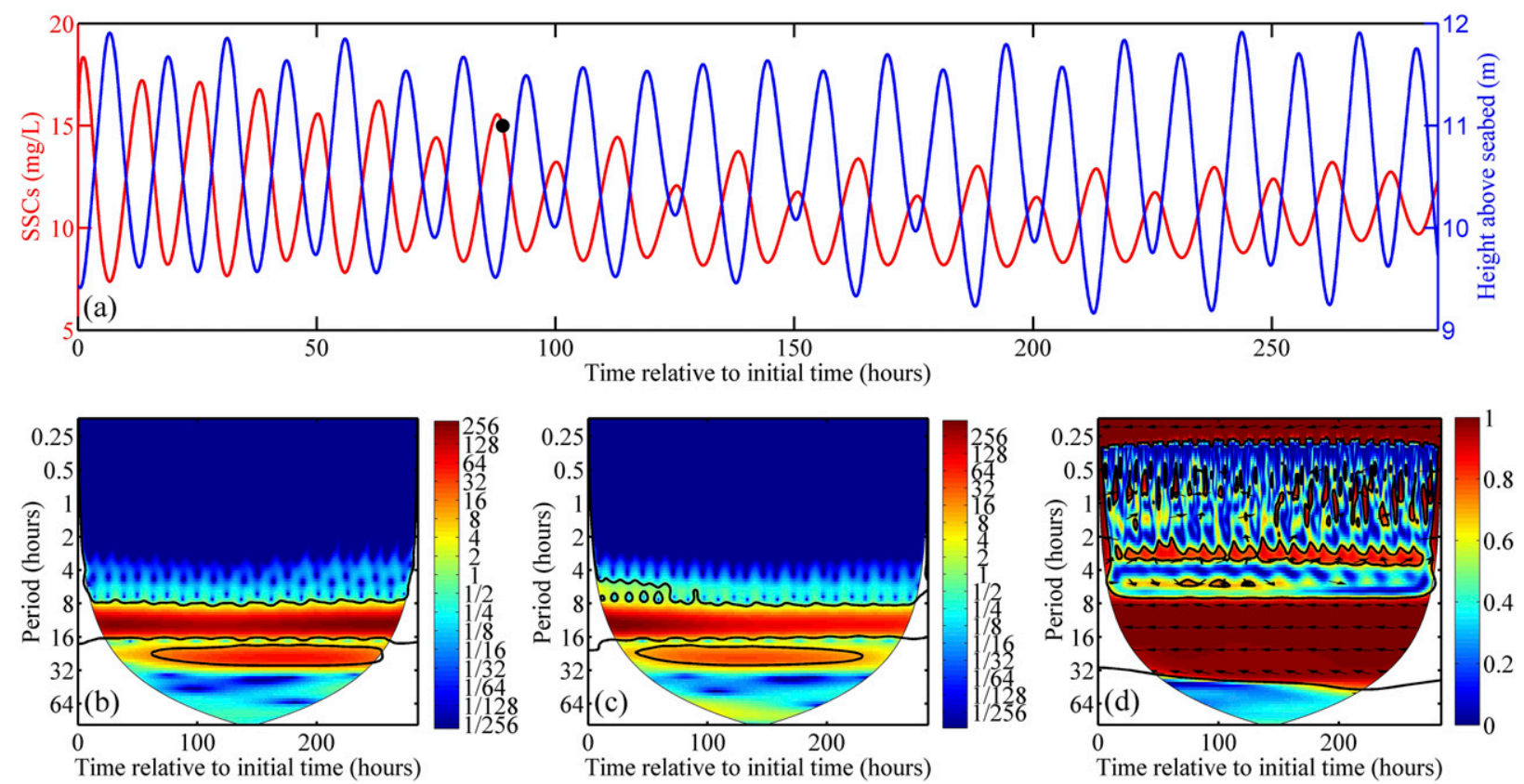

FIG. 12. (a) Interpolated surface SSCs (red line), height above seabed (blue line), and observed surface SSCs (black dot) at station 1 in PE_21. (b) The continuous wavelet power spectrum of height above the seabed. (c) As in (b), but for interpolated surface SSCs. (d) Squared wavelet coherence between height above the seabed and interpolated surface SSCs at station 1 . In (b)-(d), the 5\% significance level against red noise (thick black contour) and the cone of influence where edge effects might distort the picture (white blank) are indicated. 


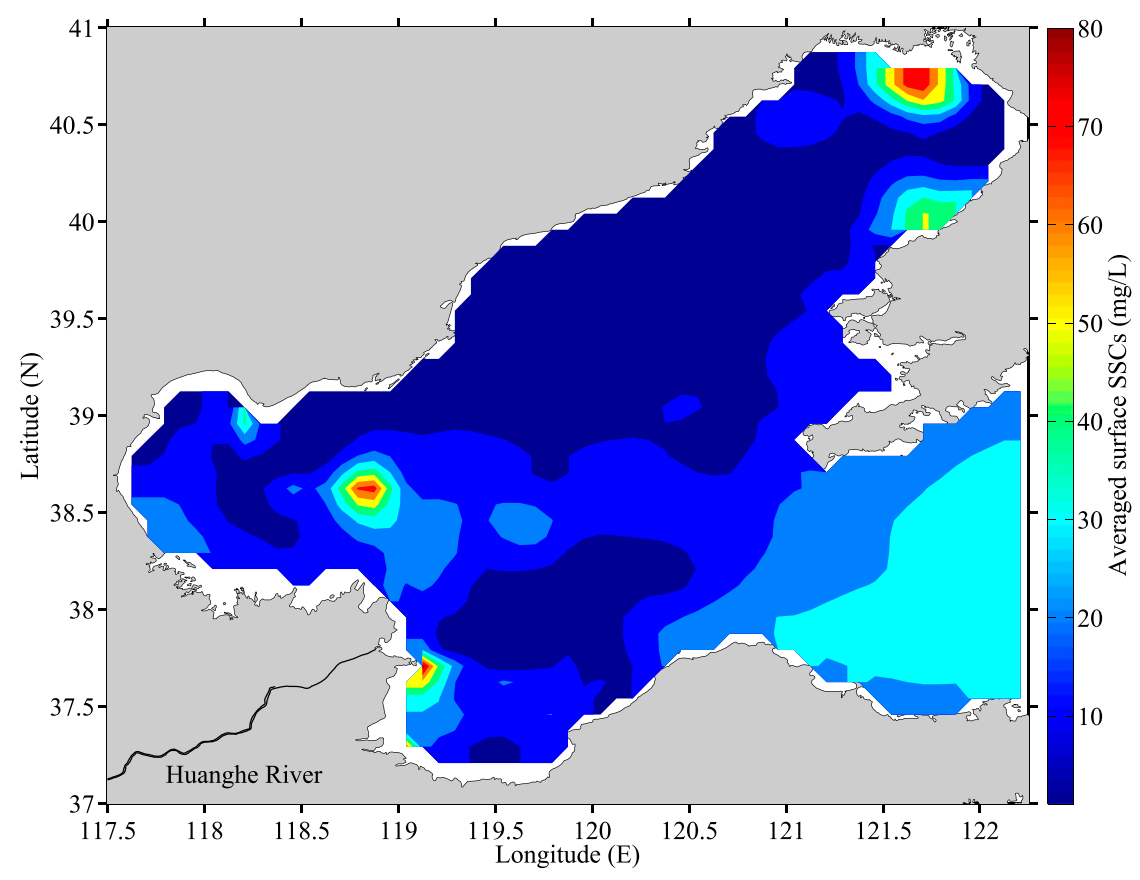

FIG. 13. The temporally averaged values of interpolated surface SSCs in PE_21.

occurred in both semidiurnal and diurnal bands (see Fig. 12d; Grinsted et al. 2004). The temporally averaged values of the final interpolated surface SSCs are shown in Fig. 13. The locations of the high values were near those shown in Fig. 2, except that near the mouth of the Huanghe River, which was formed by the inflow suspended sediments from the Huanghe River and had been shown in the remote sensing picture (Zhou et al. 2017). As the initial SSCs (Fig. 2) were obtained by directly interpolating the observations without considering the time inconsistency and there were no observations near the mouth of the Huanghe River, these high values were not shown. The aforementioned results indicated that the interpolated results obtained by the DCIM had more dynamical characteristics.

The results of practical experiments indicated that although the practical observations were sparse in both space and time, the DCIM was an effective method for interpolating these observations. In addition, with the constraints provided by the suspended sediment transport model, the final interpolated SSCs had more dynamical characteristics, which were reasonable from the viewpoint of physics.

\section{Discussions}

Through combining the dynamical constraints from the dynamical model with statistical information from observations, the DCIM can produce dynamically and statistically consistent estimates of the entire study area (Yaremchuk and Sentchev 2013). The suspended sediment transport model-that is, the dynamical model that provides the dynamical constrains and interpolates the observations in the DCIM-has been certified to be an effective model in Wang et al. (2017, 2018a,b). Besides, the adjoint data assimilation, which provides the iterative optimization in the DCIM, has rigorous mathematical basis and has matured after more than three decades of development. Therefore, the final interpolated results using the DCIM are reasonable and tested to be effective, no matter the sparse artificial observations in the twin experiments or the sparsely observed SSCs in the practical experiments.

Although the developed DCIM in this study is based on the existing suspended sediment transport model and the adjoint data assimilation scheme, which were developed in Wang et al. (2018a), there are several differences between this study and the previous studies. First, the emphases of the previous studies are placed on the parameter estimation, no matter in the twin experiments (Wang et al. 2018a) or in the practical experiments (Wang et al. 2017, 2018b); conversely, in this study the emphases are placed on the improved utilization of the sparely observed SSCs in both space and time, rather than the parameter 
estimation. Second, the adjustment strategies of the model parameters are different. In Wang et al. (2018a), the adjusted model parameters were assumed to be constant and were estimated solely. In Wang et al. (2017, 2018b), the adjusted model parameters were assumed to be spatially and temporally varying and were adjusted synchronously. Dissimilarly, the adjusted key model parameters in this study are assumed to be constant, except for the initial conditions, and are adjusted synchronously. If the iterative optimization based on the adjoint data assimilation is not implemented, the discrepancy between the interpolated results and the observations will be larger than the interpolated results using the DCIM, as shown in this study. Besides, the final interpolated results using the DCIM can involve the dynamic information, which is not included for those using the traditional interpolation methods.

Traditionally, the sparsely observed SSCs obtained from the water sampling during the large area survey are used only to calibrate other types of observations and cannot be directly used to investigate the sediment transport processes. The final interpolated SSCs using the DCIM in this study are close to the observed values and involve the dynamical information, which can be used to study the distribution and transport of the suspended sediment. However, the observed SSCs in the spatiotemporal model grids is too sparse, so the model parameters in the dynamical model are assumed to be constant to prevent the ill-posedness of the inverse problem. With the development of the satellitebased observed techniques, the satellite ocean color data have been used to map the surface SSCs in various coastal regions (e.g., Neukermans et al. 2009; Zhang et al. 2010; He et al. 2013), which can improve the spatial and temporal resolution of the SSC observations. The satellite-derived SSCs will be the potential observations to be interpolated in future studies. With the combination of the satellite-derived SSCs and the in situ observations, the interpolated results using the DCIM can be taken as the reanalysis data to further depict the distributions of the SSCs and to investigate the sediment transport processes in coastal areas. Besides, as the model parameters in the suspended sediment transport model have upper and lower limits, the constrained minimization algorithm, such as the interior penalty and barrier methods used in Zhu and Navon (1999), will be introduced into the DCIM to further improve the efficiency of the iterative optimization process in the future.

\section{Conclusions}

In this study based on the three-dimensional sigmacoordinate suspended cohesive sediment transport model with the adjoint data assimilation developed in Wang et al. (2018a), a DCIM was developed to interpolate the sparsely observed SSCs in both space and time. In the DCIM the suspended sediment transport model was taken as the dynamical constraints to interpolate the sparsely observed SSCs, and the interpolated results were optimized iteratively by adjusting the key model parameters using the adjoint method.

To show its feasibility and effectiveness, the DCIM was applied to the sparsely observed SSC observations obtained from a large area survey in the Bohai Sea. In the twin experiments, the DCIM successfully interpolated the sparse artificial SSC observations, even when the observations were heavily contaminated by data noise (see Figs. 6 and 7). In the practical experiments, the final interpolated SSCs were close to both the interpolated observations and the checked observations (see Fig. 9). Besides, when all the practical observations were interpolated, the interpolated results captured a majority (96.88\%) of observations with a factor of 2 (see Fig. 10), and the correlation coefficient between the observations and the final interpolated SSCs was 0.98. More importantly, the spatial and temporal variations of the final interpolated SSCs were reasonable from the viewpoint of physics (see Figs. 12 and 13). The aforementioned results showed that the DCIM was a feasible and effective method for interpolating the sparsely observed SSCs in both space and time, which can improve the utilization of these observations.

Acknowledgments. The authors thank the three anonymous reviewers for their constructive comments and suggestions on the earlier version of the manuscript. This work is jointly supported by the Laboratory for Regional Oceanography and Numerical Modeling of the Qingdao National Laboratory for Marine Science and Technology through Grant 2017A05; the National Key Research and Development Program of China through Grants 2016YFC1402000, 2016YFC1402304, and 2017YFC1404000; the National Natural Science Foundation of China through Grants 41630966, 41576010, and 41206001; and the Natural Science Foundation of Zhejiang Province through Grant LY15D060001. The field survey was supported by National Basic Research Program of China through Grant 2015CB453301. 


\section{REFERENCES}

Agrawal, Y., A. Whitmire, O. A. Mikkelsen, and H. Pottsmith, 2008: Light scattering by random shaped particles and consequences on measuring suspended sediments by laser diffraction. J. Geophys. Res., C04023, https://doi.org/10.1029/ 2007JC004403.

Attard, M. E., J. G. Venditti, and M. Church, 2014: Suspended sediment transport in Fraser River at Mission, British Columbia: New observations and comparison to historical records. Rev. Can. Ressour. Hydriques, 39, 356-371, https:// doi.org/10.1080/07011784.2014.942105.

Bi, N. S., Z. S. Yang, H. J. Wang, B. Q. Hu, Y. J. Ji, Z. Y. Chen, T. Yanagi, and E. Wolanski, 2010: Sediment dispersion pattern off the present Huanghe (Yellow River) subdelta and its dynamic mechanism during normal river discharge period. Estuarine Coastal Shelf Sci., 86, 352-362, https://doi.org/ 10.1016/j.ecss.2009.06.005.

Bian, C., W. Jiang, R. J. Greatbatch, and H. Ding, 2013: The suspended sediment concentration distribution in the Bohai Sea, Yellow Sea and East China Sea. J. Ocean Univ. China, 12, 345354, https://doi.org/10.1007/s11802-013-1916-3.

Black, K., and M. Rosenberg, 1994: Suspended sand measurements in a turbulent environment: Field comparison of optical and pump sampling techniques. Coastal Eng., 24, 137-150, https:// doi.org/10.1016/0378-3839(94)90030-2.

Campbell, C. G., D. T. Laycak, W. Hoppes, N. T. Tran, and F. G. Shi, 2005: High concentration suspended sediment measurements using a continuous fiber optic in-stream transmissometer. J. Hydrol., 311, 244-253, https://doi.org/10.1016/ j.jhydrol.2005.01.026.

Chen, Z., J. D. Hanson, and P. J. Curran, 1991: The form of the relationship between suspended sediment concentration and spectral reflectance: Its implications for the use of Daedalus 1268 data. Int. J. Remote Sens., 12, 215-222, https://doi.org/ 10.1080/01431169108929647.

Crickmore, M., G. Tazioli, P. Appleby, and F. Oldfield, 1990: The use of nuclear techniques in sediment transport and sedimentation problems. IHP-III Project 5.2, Technical Documents in Hydrology Series, Unesco Doc. SC-90/WS-49, 170 pp.

Egbert, G. D., and S. Y. Erofeeva, 2002: Efficient inverse modeling of barotropic ocean tides. J. Atmos. Oceanic Technol., 19, 183-204, https://doi.org/10.1175/1520-0426(2002)019<0183: EIMOBO $>2.0 . \mathrm{CO} ; 2$.

Elbern, H., A. Strunk, H. Schmidt, and O. Talagrand, 2007: Emission rate and chemical state estimation by 4-dimensional variational inversion. Atmos. Chem. Phys., 7, 3749-3769, https://doi.org/10.5194/acp-7-3749-2007.

Fan, D., J. Tu, S. Shang, and G. Cai, 2014: Characteristics of tidal-bore deposits and facies associations in the Qiantang Estuary, China. Mar. Geol., 348, 1-14, https://doi.org/ 10.1016/j.margeo.2013.11.012.

Fang, G., Y. Wang, Z. Wei, B. H. Choi, X. Wang, and J. Wang, 2004: Empirical cotidal charts of the Bohai, Yellow, and East China Seas from 10 years of TOPEX/Poseidon altimetry. J. Geophys. Res., 109, C11006, https://doi.org/10.1029/ 2004JC002484.

Gartner, J. W., 2004: Estimating suspended solids concentrations from backscatter intensity measured by acoustic Doppler current profiler in San Francisco Bay, California. Mar. Geol., 211, 169-187, https://doi.org/10.1016/j.margeo.2004.07.001.

Ge, R., H. Liu, J. Guo, Z. Zhang, and H. Wang, 2010: Synchronization approach of in-situ hydrographic observational data (in Chinese with English abstract). Adv. Mar. Sci., 28, 299-304.
Gray, J. R., and J. W. Gartner, 2009: Technological advances in suspended-sediment surrogate monitoring. Water Resour. Res., 45, W00D29, https://doi.org/10.1029/2008WR007063.

_- and M. N. Landers, 2014: Measuring suspended sediment. Status and Trends of Water Quality Worldwide, S. Ahuja S., Ed., Comprehensive Water Quality and Purification, Vol. 1, Elsevier, 157-204, https://doi.org/10.1016/B978-0-12-3821829.00012-8.

—_, G. D. Glysson, and D. S. Mueller, 2002: Comparability and accuracy of fluvial-sediment data-A view from the U.S. Geological Survey. Hydraulic Measurements and Experimental Methods 2002, T. L. Wahl et al., Eds., American Society of Civil Engineers, 6 pp., https://doi.org/10.1061/ 40655(2002)3.

Griffin, D. A., and K. R. Thompson, 1996: The adjoint method of data assimilation used operationally for shelf circulation. J. Geophys. Res., 101, 3457-3478, https://doi.org/10.1029/ 95JC03218.

Grinsted, A., J. C. Moore, and S. Jevrejeva, 2004: Application of the cross wavelet transform and wavelet coherence to geophysical time series. Nonlinear Processes Geophys., 11, 561566, https://doi.org/10.5194/npg-11-561-2004.

Guo, Z., H. Pan, W. Fan, and X. Lv, 2017: Application of surface spline interpolation in inversion of bottom friction coefficients. J. Atmos. Oceanic Technol., 34, 2021-2028, https:// doi.org/10.1175/JTECH-D-17-0012.1.

He, X., Y. Bai, D. Pan, N. Huang, X. Dong, J. Chen, C.-T. A. Chen, and Q. Cui, 2013: Using geostationary satellite ocean color data to map the diurnal dynamics of suspended particulate matter in coastal waters. Remote Sens. Environ., 133, 225-239, https://doi.org/10.1016/j.rse.2013.01.023.

Holdaway, G. P., P. D. Thorne, D. Flatt, S. E. Jones, and D. Prandle, 1999: Comparison between ADCP and transmissometer measurements of suspended sediment concentration. Cont. Shelf Res., 19, 421-441, https://doi.org/10.1016/ S0278-4343(98)00097-1.

Hoteit, I., B. Cornuelle, S. Y. Kim, G. Forget, A. Köhl, and E. Terrill, 2009: Assessing 4D-VAR for dynamical mapping of coastal high-frequency radar in San Diego. Dyn. Atmos. Oceans, 48, 175-197, https://doi.org/10.1016/ j.dynatmoce.2008.11.005.

Jia, J., Y. Wang, S. Gao, A. Wang, and Z. Li, 2006: Interpreting grain-size trends associated with bedload transport on the intertidal flats at Dafeng, central Jiangsu coast. Chin. Sci. Bull., 51, 341-351, https://doi.org/10.1007/s11434-006-0341-z.

Jiang, W., T. Pohlmann, J. Sun, and A. Starke, 2004: SPM transport in the Bohai Sea: Field experiments and numerical modelling. J. Mar. Syst., 44, 175-188, https://doi.org/10.1016/ j.jmarsys.2003.09.009.

Law, D., A. Bale, and S. Jones, 1997: Adaptation of focused beam reflectance measurement to in-situ particle sizing in estuaries and coastal waters. Mar. Geol., 140, 47-59, https://doi.org/ 10.1016/S0025-3227(97)00021-2.

Lebedev, K. V., M. Yaremchuk, H. Mitsudera, I. Nakano, and G. Yuan, 2003: Monitoring the Kuroshio Extension with dynamically constrained synthesis of the acoustic tomography, satellite altimeter and in situ data. J. Oceanogr., 59, 751-763, https://doi.org/10.1023/B:JOCE.0000009568.06949.c5.

Lee, C., D. J. Schwab, D. Beletsky, J. Stroud, and B. Lesht, 2007: Numerical modeling of mixed sediment resuspension, transport, and deposition during the March 1998 episodic events in southern Lake Michigan. J. Geophys. Res., 112, C02018, https://doi.org/10.1029/2005JC003419. 
Li, L., and P. Revesz, 2002: A comparison of spatio-temporal interpolation methods. Geographic Information Science: Second International Conference, GIScience 2002, M. J. Egenhofer and D. M. Mark, Eds., Lecture Notes in Computer Science, Vol. 2478, Springer, 145-160.

_ and — 2004: Interpolation methods for spatio-temporal geographic data. Comput. Environ. Urban Syst., 28, 201-227, https://doi.org/10.1016/S0198-9715(03)00018-8.

Liu, G., H. Wang, S. Sun, and B. Han, 2003: Numerical study on density residual currents of the Bohai Sea in summer. Chin. J. Oceanol. Limnol., 21, 106-113.

Mayer, L. M., R. G. Keil, S. A. Macko, S. B. Joye, K. C. Ruttenberg, and R. C. Aller, 1998: Importance of suspended participates in riverine delivery of bioavailable nitrogen to coastal zones. Global Biogeochem. Cycles, 12, 573-579, https://doi.org/ 10.1029/98GB02267.

Navon, I. M., 1998: Practical and theoretical aspects of adjoint parameter estimation and identifiability in meteorology and oceanography. Dyn. Atmos. Oceans, 27, 55-79, https://doi.org/ 10.1016/S0377-0265(97)00032-8.

Neukermans, G., K. Ruddick, E. Bernard, D. Ramon, B. Nechad, and P. Y. Deschamps, 2009: Mapping total suspended matter from geostationary satellites: A feasibility study with SEVIRI in the Southern North Sea. Opt. Express, 17, 14029, https://doi.org/10.1364/OE.17.014029.

Partheniades, E., 1965: Erosion and deposition of cohesive soils. J. Hydraul. Div., 91, 105-139.

Pavanelli, D., and A. Bigi, 2005: Indirect methods to estimate suspended sediment concentration: Reliability and relationship of turbidity and settleable solids. Biosyst. Eng., 90, 75-83, https://doi.org/10.1016/j.biosystemseng.2004.09.001.

Rai, A. K., and A. Kumar, 2015: Continuous measurement of suspended sediment concentration: Technological advancement and future outlook. Measurement, 76, 209-227, https:// doi.org/10.1016/j.measurement.2015.08.013.

Saha, S., and Coauthors, 2014: The NCEP Climate Forecast System Version 2. J. Climate, 27, 2185-2208, https://doi.org/10.1175/ JCLI-D-12-00823.1.

Sasaki, Y., 1970: Some basic formalisms in numerical variational analysis. Mon. Wea. Rev., 98, 875-883, https://doi.org/10.1175/ 1520-0493(1970)098<0875:SBFINV>2.3.CO;2.

Song, Y., and D. Haidvogel, 1994: A semi-implicit ocean circulation model using a generalized topography-following coordinate system. J. Comput. Phys., 115, 228-244, https:// doi.org/10.1006/jcph.1994.1189.

Sutherland, T., P. Lane, C. Amos, and J. Downing, 2000: The calibration of optical backscatter sensors for suspended sediment of varying darkness levels. Mar. Geol., 162, 587-597, https:// doi.org/10.1016/S0025-3227(99)00080-8.

Thacker, W. C., and R. B. Long, 1988: Fitting dynamics to data. J. Geophys. Res., 93, 1227-1240, https://doi.org/10.1029/ JC093iC02p01227.

Wan, X., X. Bao, D. Wu, and H. Jiang, 2004: Numerical diagnostic simulation of summertime tide-induced, wind-driven and thermohaline currents in the Bohai Sea (in Chinese with English abstract). Oceanol. Limnol. Sin., 35, 41-47.

Wang, D., J. Zhang, Y. P. Wang, X. Lv, Y. Yang, D. Fan, and S. Gao, 2017: A methodology for estimating the parameters in three-dimensional cohesive sediment transport models by assimilating in situ observations with adjoint method. J. Atmos. Oceanic Technol., 34, 1469-1482, https://doi.org/10.1175/ JTECH-D-16-0194.1.
—, A. Cao, J. Zhang, D. Fan, Y. Liu, and Y. Zhang, 2018a: A three-dimensional cohesive sediment transport model with data assimilation: Model development, sensitivity analysis and parameter estimation. Estuarine Coastal Shelf Sci., 206, 87-100, https://doi.org/10.1016/j.ecss.2016.08.027.

and Coauthors, 2018b: Parameter estimation for a cohesive sediment transport model by assimilating satellite observations in the Hangzhou Bay: Temporal variations and spatial distributions. Ocean Modell., 121, 34-48, https://doi.org/ 10.1016/j.ocemod.2017.11.007

Wang, H., A. Wang, N. Bi, X. Zeng, and H. Xiao, 2014: Seasonal distribution of suspended sediment in the Bohai Sea, China. Cont. Shelf Res., 90, 17-32, https://doi.org/10.1016/j.csr.2014.03.006.

Wilson, G. W., and A. E. Hay, 2015: Acoustic backscatter inversion for suspended sediment concentration and size: A new approach using statistical inverse theory. Cont. Shelf Res., 106, 130-139, https://doi.org/10.1016/j.csr.2015.07.005.

Wren, D. G., B. D. Barkdoll, R. A. Kuhnle, and R. W. Derrow, 2000: Field techniques for suspended-sediment measurement. J. Hydraul. Eng., 126, 97-104, https://doi.org/10.1061/(ASCE) 0733-9429(2000)126:2(97).

Yang, X., Z. Mao, H. Huang, and Q. Zhu, 2016: Using GOCI retrieval data to initialize and validate a sediment transport model for monitoring diurnal variation of SSC in Hangzhou Bay, China. Water, 8, 108, https://doi.org/10.3390/w8030108.

Yaremchuk, M., and A. Sentchev, 2013: Interpolation of the radial velocity data from coastal HF radars. Radar Systems: Technology, Principles and Applications, W.-Q. Wang, Ed., Engineering Tools, Techniques and Tables, Nova Science Publishers, 135-166.

YRCCMWRC, 2015: Yellow river sediment bulletin 2015. Yellow River Conservancy Commission of Ministry of Water Resource of China, 36 pp., http://www.yellowriver.gov.cn/ nishagonggao/2015/index.html.

Yu, L., and J. J. O'Brien, 1992: On the initial condition in parameter estimation. J. Phys. Oceanogr., 22, 1361-1364, https:// doi.org/10.1175/1520-0485(1992)022<1361:OTICIP>2.0.CO;2.

Zhang, M. W., J. W. Tang, Q. Dong, Q. T. Song, and J. Ding, 2010: Retrieval of total suspended matter concentration in the Yellow and East China Seas from MODIS imagery. Remote Sens. Environ., 114, 392-403, https://doi.org/10.1016/ j.rse.2009.09.016.

Zhang, Y., M. Bocquet, V. Mallet, C. Seigneur, and A. Baklanov, 2012: Real-time air quality forecasting, part I: History, techniques, and current status. Atmos. Environ., 60, 632-655, https://doi.org/10.1016/j.atmosenv.2012.06.031.

Zhang, Y. Y., E. R. Zhang, and J. Zhang, 2008: Modeling on adsorption-desorption of trace metals to suspended particle matter in the Changjiang Estuary. Environ. Geol., 53, 17511766, https://doi.org/10.1007/s00254-007-0781-z.

Zhou, Z., C. Bian, C. Wang, W. Jiang, and R. Bi, 2017: Quantitative assessment on multiple timescale features and dynamics of sea surface suspended sediment concentration using remote sensing data. J. Geophys. Res. Oceans, 122, 8739-8752, https:// doi.org/10.1002/2017JC013082.

Zhu, Y., and I. M. Navon, 1999: Impact of parameter estimation on the performance of the FSU global spectral model using its fullphysics adjoint. Mon. Wea. Rev., 127, 1497-1517, https://doi.org/ 10.1175/1520-0493(1999)127<1497:IOPEOT>2.0.CO;2.

Zuo, L.-q., Y.-j. Lu, Y.-p. Wang, and H.-x. Liu, 2014: Field observation and analysis of wave-current-sediment movement in Caofeidian Sea area in the Bohai Bay, China. China Ocean Eng., 28, 331-348, https://doi.org/10.1007/s13344-014-0027-2. 